\title{
Three kinds of new hybrid projection methods for a finite family of quasi-asymptotically pseudocontractive mappings in Hilbert spaces
}

\author{
Yuanxing Liu', Liguo Zheng ${ }^{2}$, Peiyuan Wang ${ }^{3,4}$ and Haiyun Zhou ${ }^{5^{*}}$
}

\author{
"Correspondence: \\ zhouhy123@aliyun.com \\ ${ }^{5}$ Department of Mathematics, \\ Shijiazhuang Mechanical \\ Engineering College, Shijiazhuang, \\ 050003, China \\ Full list of author information is \\ available at the end of the article
}

\begin{abstract}
In the present paper, we propose three kinds of new algorithms for a finite family of quasi-asymptotically pseudocontractive mappings in real Hilbert spaces. By using some new analysis techniques, we prove the strong convergence of the proposed algorithms. Some numerical examples are also included to illustrate the effectiveness of the proposed algorithms. The results presented in this paper are interesting extensions of those well-known results.

MSC: Primary 41A65; 47H17; secondary 47J20

Keywords: a finite family of quasi-asymptotically pseudocontractive mapping; uniformly L-Lipschitz mapping; iterative algorithm; strong convergence; Hilbert space
\end{abstract}

\section{Introduction}

Throughout this paper, we assume that $H$ is a real Hilbert space with inner product $\langle\cdot, \cdot\rangle$ and the induced norm $\|\cdot\|$, respectively. Let $C$ be a nonempty, closed, and convex subset of $H$ and $T: C \rightarrow C$ a self-mapping of $C$ into itself. We use Fix $(T)$ to denote the fixed point set of $T$, i.e., $\operatorname{Fix}(T)=\{x \in C: x=T x\}$.

Over the past century or so, fixed point theory of Lipschitzian and non-Lipschitzian mappings has been developed into a really important and active field of study in both pure and applied mathematics. Especially, the research on the existence and convergence of fixed points for nonexpansive mappings and pseudocontractive mappings in the framework of Hilbert and Banach spaces has made great advancements since 1965; see, for instance, $[1-3]$ and the references therein.

As generalizations of nonexpansive mappings and pseudocontractive mappings, the classes of asymptotically nonexpansive mappings and asymptotically pseudocontractive mappings were introduced by some authors, respectively; see, for instance, [4-6].

Let $E$ be a Banach space and $C$ a nonempty subset of $E$.

Recall that a mapping $T: C \rightarrow C$ is said to be asymptotically nonexpansive [4] if there exists a sequence $\left\{k_{n}\right\}$ of positive real numbers with $k_{n} \rightarrow 1$ such that

$$
\left\|T^{n} x-T^{n} y\right\| \leq k_{n}\|x-y\|
$$

(c) 2015 Liu et al. This article is distributed under the terms of the Creative Commons Attribution 4.0 International License (http://creativecommons.org/licenses/by/4.0/), which permits unrestricted use, distribution, and reproduction in any medium, provided you give appropriate credit to the original author(s) and the source, provide a link to the Creative Commons license, and indicate if changes were made. 
for all $x, y \in C$ and all $n \geq 1$.

The class of asymptotically nonexpansive mappings was introduced by Goebel and Kirk [4] in 1972. From (1.1), we know that if $T$ is nonexpansive, then it is asymptotically nonexpansive with a constant sequence $\{1\}$, but the converse may be not true in general, which can be seen from the example in [4] that is asymptotically nonexpansive but it is not nonexpansive, thus, the class of asymptotically nonexpansive mappings includes properly the class of nonexpansive mappings as a subclass. An early fundamental result, due to Goebel and Kirk [4], states that if $C$ is a nonempty, bounded, closed, and convex subset of a uniformly convex Banach space $E$, then every asymptotically nonexpansive self-mapping $T$ of $C$ has a fixed point. Further, the set $\operatorname{Fix}(T)$ of fixed points of $T$ is closed and convex. Since 1972, many authors have studied the weak and strong convergence problems of the iterative algorithms for such a class of mappings; see, for instance, [7-9] and the references therein.

The class of asymptotically pseudocontractive mappings was introduced by Schu [5] in 1991.

Recall that a mapping $T: C \rightarrow H$ is called asymptotically pseudocontractive if there exists a sequence $\left\{k_{n}\right\} \subset[1, \infty)$ with $k_{n} \rightarrow 1$ for which the following inequality holds:

$$
\left\langle T^{n} x-T^{n} y, x-y\right\rangle \leq k_{n}\|x-y\|^{2},
$$

for all $x, y \in C$ and all $n \geq 1$.

$T$ is said to be quasi-asymptotically pseudocontractive if $\operatorname{Fix}(T) \neq \emptyset$ and there exists a sequence $\left\{k_{n}\right\} \subset[1, \infty)$ with $k_{n} \rightarrow 1$ for which the following inequality holds:

$$
\left\langle T^{n} x-p, x-p\right\rangle \leq k_{n}\|x-p\|^{2},
$$

for all $x \in C, p \in \operatorname{Fix}(T)$ and all $n \geq 1$.

Without loss of generality, we can assume that $1 \leq k_{n}<2$, for all $n \geq 1$.

In 1996, Liu [6] introduced the class of $\kappa$-strictly asymptotically pseudocontractive mappings in Hilbert spaces. A mapping $T: C \rightarrow C$ is called $\kappa$-strictly asymptotically pseudocontractive if there exist some $\kappa \in[0,1)$ and some real sequence $\left\{k_{n}\right\} \subset[1, \infty)$ with $k_{n} \rightarrow 1$ such that

$$
\left\|T^{n} x-T^{n} y\right\|^{2} \leq k_{n}^{2}\|x-y\|^{2}+\kappa\left\|\left(I-T^{n}\right) x-\left(I-T^{n}\right) y\right\|^{2},
$$

for all $x, y \in C$ and all $n \geq 1$.

A mapping $T: C \rightarrow C$ is called quasi- $\kappa$-strictly asymptotically pseudocontractive if Fix $(T) \neq \emptyset$, and there exist some $\kappa \in[0,1)$ and some real sequence $\left\{k_{n}\right\} \subset[1, \infty)$ with $k_{n} \rightarrow 1$ such that

$$
\left\|T^{n} x-y\right\|^{2} \leq k_{n}^{2}\|x-y\|^{2}+\kappa\left\|\left(I-T^{n}\right) x\right\|^{2},
$$

for all $x \in C, y \in \operatorname{Fix}(T)$ and $n \geq 1$.

A mapping $T: C \rightarrow C$ is said to be uniformly $L$-Lipschtzian if there exists some $L>0$ such that

$$
\left\|T^{n} x-T^{n} y\right\| \leq L\|x-y\|
$$

for all $x, y \in C$ and for all $n \geq 1$. 
Remark 1.1 We note that every $\kappa$-strictly asymptotically pseudocontractive mapping is uniformly $L$-Lipschitzian with the Lipschitz constant $L=\frac{M+\sqrt{\kappa}}{1-\sqrt{\kappa}}$, where $M=\sup _{n}\left\{k_{n}\right\}$. In particular, every asymptotically nonexpansive mapping is uniformly $L$-Lipschitzian with $L=\sup \left\{k_{n}: n \geq 1\right\}$.

Remark 1.2 It is clear that every asymptotically nonexpansive mapping is 0 -strictly asymptotically pseudocontractive; while every asymptotically pseudocontractive mapping with sequence $\left\{k_{n}\right\}$ is 1 -strictly asymptotically pseudocontractive with sequence $\left\{2 k_{n}-1\right\}$.

Remark 1.3 It is also clear that every asymptotically pseudocontractive mapping with $\operatorname{Fix}(T) \neq \emptyset$ is quasi-asymptotically pseudocontractive, but the converse may be not true in general, which can be seen from the following counterexample.

Take $C=[0,2 \pi]$ and define a mapping $T: C \rightarrow \mathbb{R}$ by

$$
T x=\frac{2}{3} x \cos (x), \quad x \in C
$$

Then $T$ is quasi-asymptotically pseudocontractive, but it is not asymptotically pseudocontractive. Indeed, assume that $x=T x$, then $x=0$, and hence $\operatorname{Fix}(T)=\{0\}$.

For all $x \in C$, we have

$$
|T x-0|=\left|\frac{2}{3} x \cos (x)\right| \leq|x-0|
$$

which means that $T$ is quasi-nonexpansive, and hence it is quasi-asymptotically pseudocontractive. On the other hand, if we take $x=2 \pi$ and $y=\pi$, then we have

$$
\langle T x-T y, x-y\rangle=2 \pi^{2} \geq k_{1} \pi^{2}=k_{1}|x-y|^{2},
$$

which means that $T$ is not asymptotically pseudocontractive.

Remark 1.4 The class of asymptotically pseudocontractive mappings is a generalization of the class of pseudocontractive mappings, and the former contains properly the class of asymptotically nonexpansive mappings as a subclass, which can be seen from the following example.

For $x \in[0,1]$, define a mapping $T:[0,1] \rightarrow[0,1]$ by

$$
T x=\left(1-x^{\frac{2}{3}}\right)^{\frac{3}{2}}, \quad x \in[0,1]
$$

Then $T$ is asymptotically pseudocontractive but it is not asymptotically nonexpansive.

Recently, as a generalization of Haugazeau's algorithm, the so-called hybrid projection algorithm was developed rapidly for finding the nearest fixed point of certain quasinonexpansive mappings; see, for instance, Bauschke and Combettes [10] and the references therein.

By virtue of the hybrid projection methods, Nakajo and Takahashi [11] established some strong convergence results for nonexpansive mappings and nonexpansive semigroups in 
a real Hilbert space; Marino and $\mathrm{Xu}$ [12] proved a strong convergence theorem for strictpseudo-contractions in a real Hilbert space; Zhou [13] extended Marino and Xu's strong convergence theorem to the more general class of Lipschitz pseudocontractive mappings; Zhou [14] generalized and extended the main results of [13] to the class of asymptotically pseudocontractive mappings; Zhou and $\mathrm{Su}$ [15] further extended the main results in [14] to a family of uniformly $L$-Lipschitz continuous and quasi-asymptotically pseudocontractive mappings.

We observe that the construction of the half-spaces $C_{n}$ in [15] is complicated, and hence the computation of the metric projections $P_{C_{n}} x_{1}$ is difficult.

Our concern now is the following: Can one design some simple and new hybrid projection algorithms for finding a common fixed point for a finite family of quasi-asymptotically pseudocontractive mappings?

The purpose of this paper is to propose three kinds of new hybrid projection algorithms for constructing a common fixed point of a finite family of quasi-asymptotically pseudocontractive mappings in a real Hilbert space. By using some new analysis techniques, we prove the strong convergence of the proposed algorithms. Some numerical examples are also included to illustrate the effectiveness of the proposed algorithms. The results presented in this paper improve and extend the related ones obtained by some authors.

\section{Preliminaries}

For uniformly $L$-Lipschitzian mappings, the following fixed point theorem is well known; see, for example, Cassini and Maluta [16].

Theorem CM Let E be a uniformly convex Banach space with $N(E)>1, C$ be a nonempty, bounded, and closed convex subset of $E$ and $T: C \rightarrow C$ be a uniformly L-Lipschitzian mapping. If $L<\sqrt{N(E)}$, where $N(E)$ denotes the normal structure coefficient of $E$, then $T$ has a fixed point in $C$.

Remark 2.1 It is well known that $N(H)=\sqrt{2}$. Thus, in the setting of a Hilbert space $H$, every uniformly $L$-Lipschitzian mapping $T: C \rightarrow C$ from a nonempty, bounded, and closed convex subset $C$ of $H$ into itself has a fixed point in $C$ provided that $L<\sqrt[4]{2}$.

In [14], a fixed point theorem was established for asymptotically pseudocontractive mappings in Hilbert spaces.

Theorem $\mathbf{Z}$ Let $C$ be a nonempty, bounded, and closed convex subset of a real Hilbert space $H$ and $T: C \rightarrow C$ be a uniformly L-Lipschitzian and asymptotically pseudocontractive mapping which is also uniformly asymptotically regular, i.e., $\lim _{n \rightarrow \infty} \sup _{x \in C}\left\{\| T^{n+1} x-\right.$ $\left.T^{n} x \|\right\}=0$. Then $T$ has a fixed point in $C$.

Theorem $\mathrm{Z}$ is the first fixed point theorem for asymptotically pseudocontractive mappings in Hilbert spaces, which is of importance and interest.

Let $C$ be a nonempty, closed, and convex subset of a real Hilbert space $H$. For every point $x \in H$ there exists a unique nearest point in $C$, denoted by $P_{C} x$, such that

$$
\left\|x-P_{C} x\right\| \leq\|x-y\|, \quad \text { for all } y \in C,
$$


where $P_{C}$ is called the metric projection of $H$ onto $C$. We know that $P_{C}$ is a nonexpansive mapping.

The following first two lemmas are well known.

Lemma 2.1 (see, e.g., [1-3]) Let C be a nonempty, closed, and convex subset of real Hilbert space $H$. Given $x \in H$ and $z \in C$. Then $z=P_{C} x$ if and only if we have the relation

$$
\langle x-z, y-z\rangle \leq 0, \quad \text { for all } y \in C .
$$

Lemma 2.2 (see, e.g., [1-3]) Let $C$ be a nonempty closed convex subset of a real Hilbert space $H$ and $P_{C}: H \rightarrow C$ be the metric projection from $H$ onto $C$. Then the following inequality holds:

$$
\left\|y-P_{C} x\right\|^{2}+\left\|x-P_{C} x\right\|^{2} \leq\|x-y\|^{2}, \quad \forall x \in H, \forall y \in C .
$$

The next lemma is due to Zhou and Su [15]. For the sake of completeness, we include its proof here.

Lemma 2.3 Let $C$ be a nonempty, bounded, and closed convex subset of a real Hilbert space H. Let $T: C \rightarrow C$ be a uniformly L-Lipschitzian and quasi-asymptotically pseudocontractive mapping. Then $\operatorname{Fix}(T)$ is a closed convex subset of $C$.

Proof Since $T$ is uniformly $L$-Lipschitzian continuous, $\operatorname{Fix}(T)$ is closed. We need to show that $\operatorname{Fix}(T)$ is convex. To this aim, let $p_{i} \in \operatorname{Fix}(T)(i=1,2)$ and write $p=t p_{1}+(1-t) p_{2}$ for $t \in(0,1)$. We plan to show that $p=T p$. To see this, we take $\alpha \in\left(0, \frac{1}{1+L}\right)$, and define $y_{\alpha, n}=(1-$ $\alpha) p+\alpha T^{n} p$ for each $n \geq 1$. Then, in view of the quasi-asymptotic pseudocontractiveness of $T$, we have, $\forall z \in \operatorname{Fix}(T)$,

$$
\begin{aligned}
\left\|p-T^{n} p\right\|^{2}= & \left\langle p-T^{n} p, p-T^{n} p\right\rangle \\
= & \frac{1}{\alpha}\left\langle p-y_{\alpha, n}, p-T^{n} p\right\rangle \\
= & \frac{1}{\alpha}\left\langle p-y_{\alpha, n}, p-T^{n} p-\left(y_{\alpha, n}-T^{n} y_{\alpha, n}\right)\right\rangle+\frac{1}{\alpha}\left\langle p-y_{\alpha, n}, y_{\alpha, n}-T^{n} y_{\alpha, n}\right\rangle \\
\leq & \frac{1+L}{\alpha}\left\|p-y_{\alpha, n}\right\|^{2}+\frac{1}{\alpha}\left\langle p-z, y_{\alpha, n}-T^{n} y_{\alpha, n}\right\rangle \\
& +\frac{1}{\alpha}\left\langle z-y_{\alpha, n},\left(I-T^{n}\right) y_{\alpha, n}\right\rangle \\
\leq & \frac{1+L}{\alpha}\left\|p-y_{\alpha, n}\right\|^{2}+\frac{1}{\alpha}\left\langle p-z,\left(I-T^{n}\right) y_{\alpha, n}\right\rangle+\frac{1}{\alpha}\left(k_{n}-1\right)(\operatorname{diam} C)^{2} \\
= & \alpha(1+L)\left\|p-T^{n} p\right\|^{2}+\frac{1}{\alpha}\left\langle p-z,\left(I-T^{n}\right) y_{\alpha, n}\right\rangle \\
& +\frac{1}{\alpha}\left(k_{n}-1\right)(\operatorname{diam} C)^{2},
\end{aligned}
$$

from which it turns out that

$$
\alpha[1-(1+L) \alpha]\left\|p-T^{n} p\right\|^{2} \leq\left\langle p-z,\left(I-T^{n}\right) y_{\alpha, n}\right\rangle+\left(k_{n}-1\right)(\operatorname{diam} C)^{2} .
$$


Taking $z=p_{i}(i=1,2)$ in (2.4), multiplying $t$ and $(1-t)$ on both sides of (2.4), respectively, and adding up yield

$$
\alpha[1-(1+L) \alpha]\left\|p-T^{n} p\right\|^{2} \leq\left(k_{n}-1\right)(\operatorname{diam} C)^{2} .
$$

Letting $n \rightarrow \infty$ in (2.5) yields $T^{n} p \rightarrow p$. Since $T$ is continuous, we have $T^{n+1} p \rightarrow T p$ as $n \rightarrow \infty$, so that $p=T p$. This proves that $\operatorname{Fix}(T)$ is a closed convex subset of $C$.

Remark 2.2 In the proof of Lemma 2.3 above, the assumption of quasi-asymptotic pseudocontractiveness of mapping $T$ has been used.

\section{Main results}

In this section, we present three kinds of new hybrid projection algorithms for finding a common fixed point for a finite family of uniformly $L_{i}$-Lipschitzian and quasiasymptotically pseudocontractive mappings in Hilbert spaces. Let $N$ be a fixed positive integer. We put $I=\{0,1,2, \ldots, N-1\}$. For any positive integer $n$, we write $n=(h(n)-1) N+$ $i(n)$, where $h(n) \rightarrow \infty$ as $n \rightarrow \infty$ and $i(n) \in I$, for all $n \geq 0$.

First, we prove the following strong convergence theorem for a finite family of uniformly $L_{i}$-Lipschitzian and quasi-asymptotically pseudocontractive mappings in Hilbert spaces.

Theorem 3.1 Let C be a bounded, closed, and convex subset of a real Hilbert space H. Let $\left\{T_{i}\right\}_{i=0}^{N-1}: C \rightarrow C$ be a finite family of uniformly $L_{i}$-Lipschitzian and quasi-asymptotically pseudocontractive mappings such that $F=\bigcap_{i=0}^{N-1} \operatorname{Fix}\left(T_{i}\right) \neq \emptyset$. Assume the control sequence $\left\{\alpha_{n}\right\}$ is chosen so that $\alpha_{n} \in[a, b]$ for some $a, b \in\left(0, \frac{1}{1+L}\right)$, where $L=\max \left\{L_{i}: 0 \leq i \leq N-1\right\}$. Let a sequence $\left\{x_{n}\right\}$ be generated by the following manner:

$$
\left\{\begin{array}{l}
x_{0} \in C \quad \text { chosen arbitrarily, } \\
y_{n}=\left(1-\alpha_{n}\right) x_{n}+\alpha_{n} T_{i(n)}^{h(n)} x_{n}, \quad n \geq 0, \\
C_{n}=\left\{z \in C: \alpha_{n}\left[1-(1+L) \alpha_{n}\right]\left\|x_{n}-T_{i(n)}^{h(n)} x_{n}\right\|^{2}\right. \\
\left.\quad \leq\left\langle x_{n}-z,\left(y_{n}-T_{i(n)}^{h(n)} y_{n}\right)\right\rangle+\left(k_{h(n)}-1\right)(\operatorname{diam} C)^{2}\right\}, \quad n \geq 0, \\
Q_{0}=C \\
Q_{n}=\left\{z \in Q_{n-1}:\left\langle z-x_{n}, x_{0}-x_{n}\right\rangle \leq 0\right\}, \quad n \geq 1, \\
x_{n+1}=P_{C_{n} \cap Q_{n} x_{0}, \quad n \geq 0,}
\end{array}\right.
$$

where $k_{h(n)}=\max \left\{k_{h(n), i(n)}: 0 \leq i(n) \leq N-1\right\}$ and $k_{h(n), i(n)}$ are asymptotic sequences for $\left\{T_{i}\right\}_{i=0}^{N-1}$. Then the sequence $\left\{x_{n}\right\}$ generated by (3.1) converges strongly to $P_{F} x_{0}$.

Proof We split the proof into ten steps.

Step 1 . Show that $P_{F} x_{0}$ is well defined for every $x_{0} \in C$.

By Lemma 2.3, we know that Fix $\left(T_{i}\right)$ is a closed convex subset of $C$ for every $i \in I$. Hence, $F=\bigcap_{i=0}^{N-1} \operatorname{Fix}\left(T_{i}\right)$ is a nonempty, closed, and convex subset of $C$, consequently, $P_{F} x_{0}$ is well defined for every $x_{0} \in C$.

Step 2. Show that both $C_{n}$ and $Q_{n}$ are closed and convex, for all $n \geq 0$. This follows from the constructions of $C_{n}$ and $Q_{n}$. We omit the details.

Step 3. Show that

$$
F \subset C_{n} \cap Q_{n}, \quad \text { for all } n \geq 0 \text {. }
$$


To this aim, we prove first that $F \subset C_{n}$, for all $n \geq 0$.

Using (3.2), the uniform $L_{i}$-Lipschitz continuity of $T_{i}$ and quasi-asymptotic pseudocontractiveness of $T_{i}$, we obtain, for any $z \in F$,

$$
\begin{aligned}
\left\|x_{n}-T_{i(n)}^{h(n)} x_{n}\right\|^{2}= & \left\langle x_{n}-T_{i(n)}^{h(n)} x_{n}, x_{n}-T_{i(n)}^{h(n)} x_{n}\right\rangle \\
= & \frac{1}{\alpha_{n}}\left\langle x_{n}-y_{n}, x_{n}-T_{i(n)}^{h(n)} x_{n}\right\rangle \\
= & \frac{1}{\alpha_{n}}\left\langle x_{n}-y_{n},\left(I-T_{i(n)}^{h(n)}\right) x_{n}-\left(I-T_{i(n)}^{h(n)}\right) y_{n}\right\rangle \\
& +\frac{1}{\alpha_{n}}\left\langle x_{n}-y_{n},\left(I-T_{i(n)}^{h(n)}\right) y_{n}\right\rangle \\
\leq & \frac{1+L}{\alpha_{n}}\left\|x_{n}-y_{n}\right\|^{2}+\frac{1}{\alpha_{n}}\left\langle x_{n}-z,\left(I-T_{i(n)}^{h(n)}\right) y_{n}\right\rangle \\
& +\frac{1}{\alpha_{n}}\left(k_{h(n)}-1\right)(\operatorname{diam} C)^{2} \\
= & (1+L) \alpha_{n}\left\|x_{n}-T^{n} x_{n}\right\|^{2}+\frac{1}{\alpha_{n}}\left\langle x_{n}-z,\left(I-T_{i(n)}^{h(n)}\right) y_{n}\right\rangle \\
& +\frac{1}{\alpha_{n}}\left(k_{h(n)}-1\right)(\operatorname{diam} C)^{2},
\end{aligned}
$$

from which it turns out that

$$
\alpha_{n}\left[1-(1+L) \alpha_{n}\right]\left\|x_{n}-T_{i(n)}^{h(n)} x_{n}\right\|^{2} \leq\left\langle x_{n}-z,\left(I-T_{i(n)}^{h(n)}\right) y_{n}\right\rangle+\left(k_{h(n)}-1\right)(\operatorname{diam} C)^{2},
$$

which shows that $z \in C_{n}$, for all $n \geq 0$. This proves that $F \subset C_{n}$, for all $n \geq 0$.

As shown in Marino and Xu [12], by a simple induction, we can show that

$$
F \subset Q_{n}, \quad \text { for all } n \geq 0 \text {. }
$$

Because this is routine, we omit the details. We have shown that (3.2) holds. Hence $P_{C_{n} \cap Q_{n}} x_{0}$ is well defined. Consequently, the iteration algorithm (3.1) is well defined.

Step 4 . Show that $\lim _{n \rightarrow \infty}\left\|x_{n}-x_{0}\right\|$ exists.

In view of (3.1) and Lemma 2.1, we have $x_{n}=P_{Q_{n}} x_{0}$ and $x_{n+1} \in Q_{n}$, which means that $\left\|x_{n}-x_{0}\right\| \leq\left\|x_{n+1}-x_{0}\right\|$, for all $n \geq 0$. As $z \in F \subset Q_{n}$, we have also $\left\|x_{n}-x_{0}\right\| \leq\left\|z-x_{0}\right\|$, consequently, $\lim _{n \rightarrow \infty}\left\|x_{n}-x_{0}\right\|$ exists.

Step 5. Show that $x_{n+1}-x_{n} \rightarrow 0$ as $n \rightarrow \infty$.

By using Lemma 2.2, we have

$$
\left\|x_{n+1}-x_{n}\right\|^{2} \leq\left\|x_{n+1}-x_{0}\right\|^{2}-\left\|x_{n}-x_{0}\right\|^{2} \rightarrow 0
$$

as $n \rightarrow \infty$.

Step 6. Show that $x_{n}-T_{i(n)}^{h(n)} x_{n} \rightarrow 0$ as $n \rightarrow \infty$.

It follows from Step 5 that $x_{n+1}-x_{n} \rightarrow 0$ as $n \rightarrow \infty$. Since $x_{n+1} \in C_{n}$, noting that $\alpha_{n} \in$ $[a, b]$ for $a, b \in\left(0, \frac{1}{1+L}\right),\left\{y_{n}\right\}$ and $\left\{T_{i(n)}^{h(n)} y_{n}\right\}$ are all bounded, from the definition of $C_{n}$, we have $x_{n}-T_{i(n)}^{h(n)} x_{n} \rightarrow 0$ as $n \rightarrow \infty$.

Step 7. Show that $x_{n}-T_{i(n)} x_{n} \rightarrow 0$ as $n \rightarrow \infty$. 
Since $n=(h(n)-1)+i(n)$, we have

$$
n-N=(h(n)-1-1) N+i(n)
$$

On the other hand, since $n-N=(h(n-N)-1) N+i(n-N)$, we have $h(n)-1=h(n-N)$ and $i(n)=i(n-N)$. Observe that

$$
\begin{aligned}
\left\|x_{n}-T_{i(n)} x_{n}\right\| \leq & \left\|x_{n}-T_{i(n)}^{h(n)} x_{n}\right\|+\left\|T_{i(n)}^{h(n)} x_{n}-T_{i(n)} x_{n}\right\| \\
\leq & \left\|x_{n}-T_{i(n)}^{h(n)} x_{n}\right\|+L\left\|T_{i(n)}^{h(n)-1} x_{n}-x_{n}\right\| \\
\leq & \left\|x_{n}-T_{i(n)}^{h(n)} x_{n}\right\|+L\left\|T_{i(n)}^{h(n-N)} x_{n}-T_{i(n-N)}^{h(n-N)} x_{n-N}\right\| \\
& \quad+\left\|T_{i(n-N)}^{h(n-N)} x_{n-N}-x_{n-N}\right\|+\left\|x_{n-N}-x_{n}\right\| \\
\leq & \left\|x_{n}-T_{i(n)}^{h(n)} x_{n}\right\|+\left(1+L^{2}\right)\left\|x_{n-N}-x_{n}\right\|+\left\|T_{i(n-N)}^{h(n-N)} x_{n-N}-x_{n-N}\right\|,
\end{aligned}
$$

from which it turns out that $x_{n}-T_{i(n)} x_{n} \rightarrow 0$ as $n \rightarrow \infty$ in view of Steps 5 and 6.

Step 8 . Show that $\forall j \in I, x_{n}-T_{i(n)+j} x_{n} \rightarrow 0$ as $n \rightarrow \infty$.

Observing that

$$
\begin{aligned}
\left\|x_{n}-T_{i(n)+j} x_{n}\right\| \leq & \left\|x_{n}-x_{n+j}\right\|+\left\|x_{n+j}-T_{i(n)+j} x_{n+j}\right\| \\
& \quad+\left\|T_{i(n)+j} x_{n+j}-T_{i(n)+j} x_{n}\right\| \\
\leq & \left\|x_{n}-x_{n+j}\right\|+\left\|x_{n+j}-T_{i(n+j)} x_{n+j}\right\|+L\left\|x_{n+j}-x_{n}\right\| \\
= & (1+L)\left\|x_{n}-x_{n+j}\right\|+\left\|x_{n+j}-T_{i(n+j)} x_{n+j}\right\|,
\end{aligned}
$$

by using Steps 5 and 7, we reach the desired conclusion.

Step 9 . Show that $\forall l \in I, x_{n}-T_{l} x_{n} \rightarrow 0$ as $n \rightarrow \infty$.

Indeed, for arbitrary given $l \in I$, we can choose $j \in I$ such that $j=l-i(n)$ if $l \geq i(n)$ and $j=N+l-i(n)$ if $l<i(n)$. Then, we have $l=i(n+j)=i(n)+j$, for all $n \geq 0$. In view of Step 8, we obtain $x_{n}-T_{l} x_{n}=x_{n}-T_{i(n+j)} x_{n}=x_{n}-T_{i(n)+j} x_{n} \rightarrow 0$ as $n \rightarrow \infty$.

Step 10. Show that $x_{n} \rightarrow p$, where $p=P_{F} x_{0}$.

For $m>n$, by the definition of $Q_{n}$, we see that $Q_{m} \subset Q_{n}$. Noting that $x_{m}=P_{Q_{m}} x_{0}$ and $x_{n}=P_{Q_{n}} x_{0}$, by Lemma 2.3 , we conclude that

$$
\left\|x_{m}-x_{n}\right\|^{2} \leq\left\|x_{m}-x_{0}\right\|^{2}-\left\|x_{n}-x_{0}\right\|^{2}
$$

In view of Step 4, we deduce that $x_{m}-x_{n} \rightarrow 0$ as $m, n \rightarrow \infty$, that is, $\left\{x_{n}\right\}$ is Cauchy. Since $H$ is complete and $C$ is closed, we can assume that $x_{n} \rightarrow p \in C$ as $n \rightarrow \infty$. It follows from Step 9 that $p \in F$. From Step 2, we know that $F \subset Q_{n}$, for all $n \geq 0$. Hence, for arbitrary $z \in F$, we have

$$
\left\langle z-x_{n}, x_{0}-x_{n}\right\rangle \leq 0
$$

This leads to

$$
\left\langle z-p, x_{0}-p\right\rangle \leq 0
$$

for all $z \in F$. By Lemma 2.1, we conclude that $p=P_{F} x_{0}$. This completes the proof. 
Remark 3.1 In contrast to [15], the main difference with the paper [15] consists in the fact that the sequence $\left\{y_{n}\right\}$ in algorithm (3.1) is globally unique for the whole family of $\left\{T_{i}\right\}_{i=0}^{N-1}$.

Remark 3.2 In the proof of Theorem 3.1, the third step is really key. The assumption of quasi-asymptotic pseudocontractiveness of the mappings $\left\{T_{i}\right\}_{i=0}^{N-1}$ has been used.

Next, we consider a simpler algorithm for a finite family of uniformly $L_{i}$-Lipschitzian and quasi-asymptotically pseudocontractive mappings in real Hilbert spaces.

Theorem 3.2 Let C be a bounded, closed, and convex subset of a real Hilbert space H. Let $\left\{T_{i}\right\}_{i=0}^{N-1}: C \rightarrow C$ be a finite family of uniformly $L_{i}$-Lipschitzian and quasi-asymptotically pseudocontractive mappings such that $F=\bigcap_{i=0}^{N-1} \operatorname{Fix}\left(T_{i}\right) \neq \emptyset$. Assume the control sequence $\left\{\alpha_{n}\right\}$ is chosen so that $\alpha_{n} \in[a, b]$ for some $a, b \in\left(0, \frac{1}{1+L}\right)$, where $L=\max \left\{L_{i}: 0 \leq i \leq N-1\right\}$. Let a sequence $\left\{x_{n}\right\}$ be generated in the following manner:

$$
\left\{\begin{array}{l}
x_{0} \in H \quad \text { chosen arbitrarily, } \\
C_{1}=C, \quad x_{1}=P_{C_{1}} x_{0}, \\
y_{n}=\left(1-\alpha_{n}\right) x_{n}+\alpha_{n} T_{i(n)}^{h(n)} x_{n}, \quad n \geq 1, \\
C_{n+1}=\left\{z \in C_{n}: \alpha_{n}\left[1-(1+L) \alpha_{n}\right]\left\|x_{n}-T_{i(n)}^{h(n)} x_{n}\right\|^{2}\right. \\
\left.\quad \leq\left\langle x_{n}-z,\left(y_{n}-T_{i(n)}^{h(n)} y_{n}\right)\right\rangle+\left(k_{h(n)}-1\right)(\operatorname{diam} C)^{2}\right\}, \quad n \geq 0, \\
x_{n+1}=P_{C_{n+1}} x_{0}, \quad n \geq 1,
\end{array}\right.
$$

where $k_{h(n)}=\max \left\{k_{h(n), i(n)}: 0 \leq i(n) \leq N-1\right\}$ and $k_{h(n), i(n)}$ are asymptotic sequences for $\left\{T_{i}\right\}_{i=0}^{N-1}$. Then the sequence $\left\{x_{n}\right\}$ generated by (3.5) converges strongly to $P_{F} x_{0}$.

Proof Following the proof lines of Theorem 3.1, we can show the following.

(1) $F$ is a nonempty closed and convex subset of $C$, and hence $P_{F} x_{0}$ is well defined for every $x_{0} \in H$.

(2) $C_{n}$ is closed convex and $F \subset C_{n}$ for every $n \geq 1$.

In fact, for $n=1, C_{1}=C$ is closed convex. Assume that $C_{n}$ is closed convex for some $n \geq 1$; from the definition $C_{n+1}$, we know that $C_{n+1}$ is also closed convex for the same $n \geq 1$, and hence $C_{n}$ is closed convex for every $n \geq 1$. For $n=1, F \subset C_{1}=C$. Assume that $F \subset C_{n}$ for some $n \geq 1$; from the induction assumption, (3.3), and the definition of $C_{n+1}$, we conclude that $F \subset C_{n+1}$, and hence $F \subset C_{n}$, for all $n \geq 1$.

(3) $\lim _{n \rightarrow \infty}\left\|x_{n}-x_{0}\right\|$ exists.

In view of (3.5), we have $x_{n}=P_{C_{n}} x_{0}$. Since $C_{n+1} \subset C_{n}$ and $x_{n+1} \in C_{n+1}$, for all $n \geq 1$, we have

$$
\left\|x_{n}-x_{0}\right\| \leq\left\|x_{n+1}-x_{0}\right\|, \quad \forall n \geq 1 .
$$

On the other hand, as $F \subset C_{n}$ by (2), it follows that

$$
\left\|x_{n}-x_{0}\right\| \leq\left\|z-x_{0}\right\|, \quad \forall z \in F, \forall n \geq 1 .
$$

Combining (3.6) and (3.7), we see that $\lim _{n \rightarrow \infty}\left\|x_{n}-x_{0}\right\|$ exists.

(4) $\left\{x_{n}\right\}$ is a Cauchy sequence in $C$. 
For $m>n \geq 1$, we have $x_{m}=P_{C_{m}} x_{0} \in C_{m} \subset C_{n}$. By Lemma 2.2, we have

$$
\left\|x_{m}-x_{n}\right\|^{2} \leq\left\|x_{m}-x_{0}\right\|^{2}-\left\|x_{n}-x_{0}\right\|^{2}
$$

Letting $m, n \rightarrow \infty$ and taking the limit in (3.8), we get $x_{m}-x_{n} \rightarrow 0$ as $m, n \rightarrow \infty$, which proves that $\left\{x_{n}\right\}$ is Cauchy. We assume that $x_{n} \rightarrow p \in C$. The remainder of the proof follows exactly from Steps 5-10 in Theorem 3.1. This completes the proof.

Remark 3.3 Algorithm (3.5) is simpler than algorithm (3.1). Also, the sequence $\left\{y_{n}\right\}$ in algorithm (3.5) is globally unique for the whole family of $\left\{T_{i}\right\}_{i=0}^{N-1}$.

Finally, we present another kind of iterative algorithm for a finite family of quasiasymptotically pseudocontractive mappings in real Hilbert spaces.

Theorem 3.3 Let $C$ be a bounded and closed convex subset of a real Hilbert space H. Let $\left\{T_{i}\right\}_{i=0}^{N-1}: C \rightarrow C$ be a finite family of uniformly $L_{i}$-Lipschitzian and quasi-asymptotically pseudocontractive mappings such that $F=\bigcap_{i=0}^{N-1} \operatorname{Fix}\left(T_{i}\right) \neq \emptyset$. Assume the control sequence $\left\{\alpha_{n}\right\}$ is chosen so that $\alpha_{n} \in[a, b]$ for some $a, b \in\left(0, \frac{1}{1+L}\right)$, where $L=\max \left\{L_{i}: 0 \leq i \leq N-1\right\}$. Let a sequence $\left\{x_{n}\right\}$ be generated in the following manner:

$$
\left\{\begin{array}{l}
x_{0} \in H \quad \text { chosen arbitrarily, } \\
C_{1}=C, \quad x_{1}=P_{C_{1}} x_{0}, \\
y_{n, i}=\left(1-\alpha_{n}\right) x_{n}+\alpha_{n} T_{i}^{n} x_{n}, \quad n \geq 1, i \in I, \\
C_{n+1}=\left\{z \in C_{n}: \alpha_{n}\left[1-(1+L) \alpha_{n}\right] \sum_{i=0}^{N-1}\left\|x_{n}-T_{i}^{n} x_{n}\right\|^{2}\right. \\
\left.\quad \leq\left\langle x_{n}-z, \sum_{i=0}^{N-1}\left(y_{n, i}-T_{i}^{n} y_{n, i}\right)\right\rangle+\sum_{i=0}^{N-1}\left(k_{n, i}-1\right)(\operatorname{diam} C)^{2}\right\}, \quad n \geq 0, \\
x_{n+1}=P_{C_{n+1}} x_{0}, \quad n \geq 1,
\end{array}\right.
$$

where $k_{n, i}$ are asymptotic sequences for $\left\{T_{i}\right\}_{i=0}^{N-1}$. Then the sequence $\left\{x_{n}\right\}$ generated by (3.9) converges strongly to $P_{F} x_{0}$.

Proof As shown in Theorem 3.2, we easily show that $P_{F} x_{0}$ is well defined for every $x_{0} \in H$, $C_{n}$ is closed convex and $F \subset C_{n}$ for every $n \geq 1$. Thus, $\left\{x_{n}\right\}$ is well defined, for all $n \geq 1$. Further, $\left\{x_{n}\right\}$ is a Cauchy sequence in $C$. Therefore, $x_{n} \rightarrow p \in C$ as $n \rightarrow \infty$. In particular, we have $x_{n+1}-x_{n} \rightarrow 0$ as $n \rightarrow \infty$. Since $0<a \leq \alpha_{n} \leq b<\frac{1}{1+L}, x_{n+1} \in C_{n+1},\left\{\sum_{i=0}^{N-1}\left\|\left(I-T_{i}^{n}\right) y_{n, i}\right\|\right\}$ is bounded and $\sum_{i=0}^{N-1}\left(k_{n, i}-1\right) \rightarrow 0$, from the definition of $C_{n+1}$, we see that $x_{n}-T_{i}^{n} x_{n} \rightarrow 0$ as $n \rightarrow \infty$, for all $i \in I$. Observe that

$$
\begin{aligned}
\left\|x_{n+1}-T_{i} x_{n+1}\right\| \leq & \left\|x_{n+1}-T_{i}^{n+1} x_{n+1}\right\|+\left\|T_{i}^{n+1} x_{n+1}-T_{i}^{n+1} x_{n}\right\| \\
& +\left\|T_{i}^{n+1} x_{n}-T_{i} x_{n}\right\|+\left\|T_{i} x_{n}-T_{i} x_{n+1}\right\| \\
\leq & \left\|x_{n+1}-T_{i}^{n+1} x_{n+1}\right\|+2 L\left\|x_{n+1}-x_{n}\right\|+L\left\|x_{n}-T^{n} x_{n}\right\|,
\end{aligned}
$$

so that $x_{n}-T_{i} x_{n} \rightarrow 0$ as $n \rightarrow \infty$, for all $i \in I$. Since $x_{n} \rightarrow p$, we have $p=T_{i} p$, for all $i \in I$ and hence $p \in F$. The remainder of the proof follows exactly from Step 10 of Theorem 3.1. This completes the proof.

Remark 3.4 Algorithm (3.9) used in Theorem 3.3 is different from the ones existing in literature. 
Remark 3.5 It is interesting to extend the algorithms of this paper to an infinite family of quasi-asymptotically pseudocontractive mappings.

Remark 3.6 The work related to other iterative methods for asymptotically pseudocontractive mappings can be found in [17-21].

\section{Numerical experiments}

In this section, we provide some numerical experiments to show our algorithms are effective. In our numerical experiments, we consider the case of $N=2$. We take $T_{0}=I$, the identity mapping on $\mathbb{R}$, and use the example given in Remark 1.3 as $T_{1}$. For such a family $\left\{T_{i}\right\}_{i=0}^{1}$, we have $L_{0}=1$ and $L_{1}=\frac{2+4 \pi}{3}$, therefore, $L=\frac{2+4 \pi}{3}$. It is easy to see that $k_{h(n)}=1$, for all $n \geq 0$. Moreover, we know also that $F=\bigcap_{i=0}^{1} \operatorname{Fix}\left(T_{i}\right)=\{0\} \neq \emptyset$. We take $\alpha_{n}=\frac{1}{n+56}+\frac{1}{2+L}$, for all $n \geq 0$. For algorithms (3.1), (3.5), and (3.9), each of them iterates 70 steps.

Firstly, for algorithm (3.1), we choose $x_{0} \in[0,2 \pi]$ arbitrarily, then for 51 different initial values, we can see all the results are convergent in Figure 1.

Secondly, for algorithm (3.5), we choose $x_{0} \in[-2,10]$ arbitrarily, then for 61 different initial values, we can see all the results are convergent in Figure 2.

Finally, for algorithm (3.9), we also choose $x_{0} \in[-2,10]$ arbitrarily, then for 61 different initial values, we can also see all the results are convergent in Figure 3.
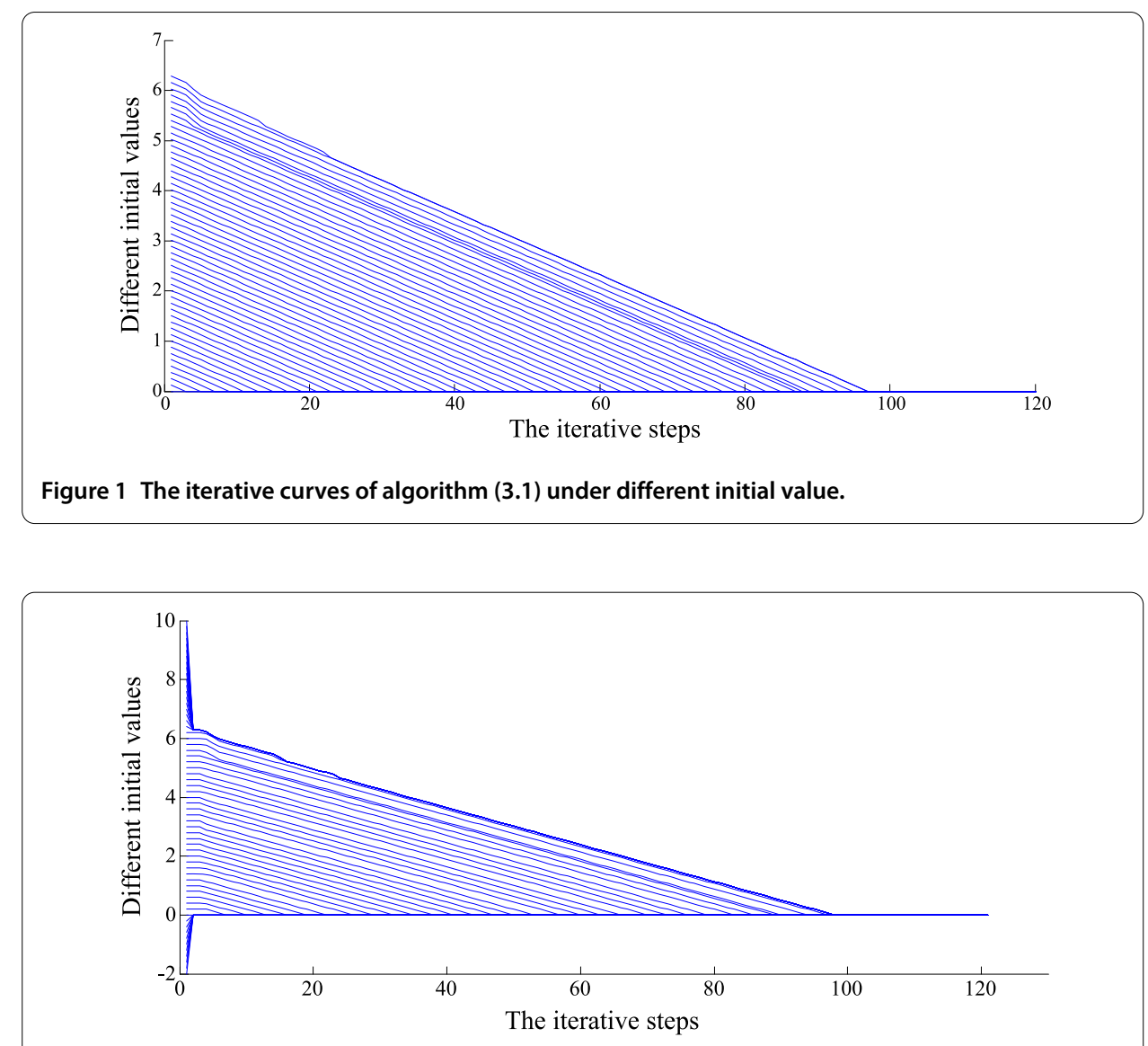

Figure 2 The iterative curves of algorithm (3.5) under different initial values. 


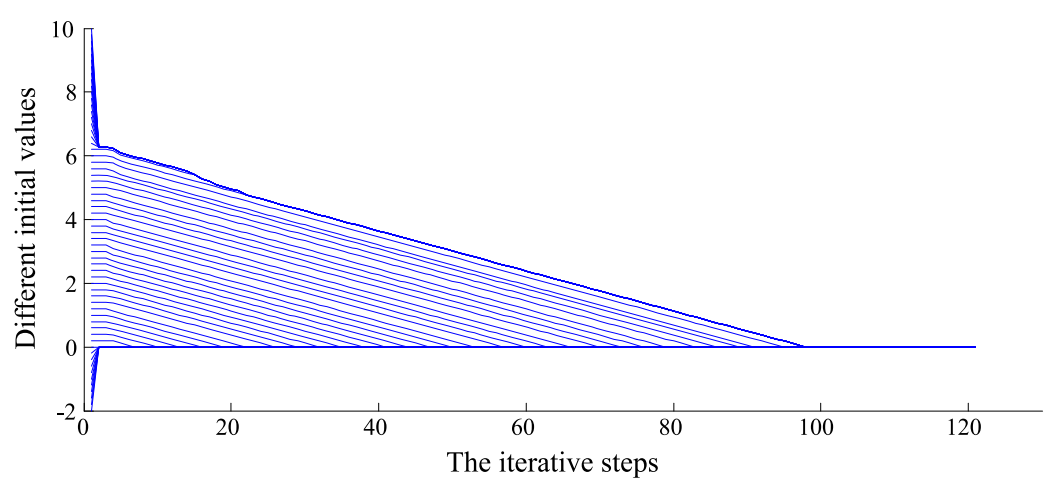

Figure 3 The iterative curves of algorithm (3.9) under different initial values.

In addition, for Figures 1, 2, and 3, we can also find that the algorithms need more iterative steps with the nonnegative initial value becoming larger in the majority situation.

\section{Conclusion}

This work contains our dedicated study aimed to develop and complement hybrid projection algorithms for finding the common fixed points of a finite family of quasiasymptotically pseudocontractive mappings in Hilbert spaces. We introduced three kinds of new hybrid projection algorithms for this class of problems, and we have proven their strong convergence. Numerical examples have been given to illustrate the effectiveness of the proposed algorithms. The results presented in the paper are a generalization and complement of the well-known ones existing in the literature.

\section{Competing interests}

The authors declare that they have no competing interests.

\section{Authors' contributions}

All authors contributed equally and significantly in writing this paper. All authors read and approved the final manuscript.

\section{Author details}

${ }^{1}$ College of Mathematics and Statistics, Hebei University of Economics and Business, Shijiazhuang, 050061, China. ${ }^{2}$ Department of Mathematics and Information, Langfang Normal University, Langfang, 065000, China. ${ }^{3}$ Postdoctoral Workstation, Naval Equipment Institute, Beijing, 102249, China. ${ }^{4}$ Naval Aviation Institution, Huludao, 125001, China. ${ }^{5}$ Department of Mathematics, Shijiazhuang Mechanical Engineering College, Shijiazhuang, 050003, China.

\section{Acknowledgements}

This research was supported by the National Natural Science Foundation of China (11071053) and Key Project of Science and Research of Hebei University of Economics and Business (2015KYZ03).

Received: 25 February 2015 Accepted: 29 June 2015 Published online: 16 July 2015

\section{References}

1. Goebel, K, Kirk, WA: Topics in Metric Fixed Point Theory. Cambridge Studies in Advanced Mathematics, vol. 28. Cambridge University Press, Cambridge (1990)

2. Chidume, CE: Geometric Properties of Banach Spaces and Nonlinear Iterations. Springer, Berlin (2008)

3. Agarwal, RP, O'Regan, D, Sahu, DR: Fixed Point Theory for Lipschitzian-Type Mappings with Applications. Springer, Berlin (2009)

4. Goebel, K, Kirk, WA: A fixed point theorem for asymptotically nonexpansive mappings. Proc. Am. Math. Soc. 35, 171-174 (1972)

5. Schu, J: Iteration construction of fixed points of asymptotically nonexpansive mappings. J. Math. Anal. Appl. 158, 407-413 (1991)

6. Liu, QH: Convergence theorems of the sequence of iterates for asymptotically demicontractive and hemicontractive mappings. Nonlinear Anal. 26, 1835-1842 (1996)

7. Cho, YJ, Zhou, H, Guo, G: Weak and strong convergence theorems for three-step iterations with errors for asymptotically nonexpansive mappings. Comput. Math. Appl. 47, 707-717 (2004) 
8. Kim, TH, Xu, HK: Strong convergence of modified Mann iterations for asymptotically nonexpansive mappings and semigroups. Nonlinear Anal. 64, 1140-1152 (2006)

9. Chidume, $C E$, Ali, B: Weak and strong convergence theorems for finite families of asymptotically nonexpansive mappings in Banach spaces. J. Math. Anal. Appl. 330, 377-387 (2007)

10. Bauschke, HH, Combettes, PL: A weak-to-strong convergence principle for Fejér-monotone methods in Hilbert space. Math. Oper. Res. 26, 248-264 (2001)

11. Nakajo, K, Takahashi, W: Strong convergence theorems for nonexpansive mappings and nonexpansive semigroups. J. Math. Anal. Appl. 279, 372-379 (2003)

12. Marino, G, Xu, HK: Weak and strong convergence theorems for strict pseudo-contractions in Hilbert spaces. J. Math. Anal. Appl. 329, 336-349 (2007)

13. Zhou, HY: Convergence theorems of fixed points for Lipschitz pseudo-contractions in Hilbert spaces. J. Math. Anal. Appl. 343, 546-556 (2008)

14. Zhou, HY: Demiclosedness principle with applications for asymptotically pseudo-contractions in Hilbert spaces. Nonlinear Anal. 70, 3140-3145 (2009)

15. Zhou, HY, Su, YF: Strong convergence theorems for a family of quasi-asymptotic pseudo-contractions in Hilbert spaces. Nonlinear Anal. 70, 4047-4052 (2009)

16. Cassini, E, Maluta, E: Fixed points for uniformly Lipschitzian mappings in spaces with uniformly normal structure. Nonlinear Anal. 9, 103-108 (1985)

17. Chidume, CE, Zegeye, H: Approximate fixed point sequences and convergence theorems for asymptotically pseudocontractive mappings. J. Math. Anal. Appl. 278, 354-366 (2003)

18. Zhou, HY: Weak convergence theorems for strict pseudo-contractions in Banach spaces. Acta Math. Sin. Engl. Ser. 30, 755-766 (2014)

19. Zhou, HY: A new iteration method for variational inequalities on the set of common fixed points for a finite family of quasi-pseudo-contractions in Hilbert spaces. J. Inequal. Appl. 2014, 218 (2014)

20. Zegeye, $\mathrm{H}$, Shahzad, $\mathrm{N}$ : An algorithm for a common fixed point of a family of pseudocontractive mappings. Fixed Point Theory Appl. 2013, 234 (2013)

21. Yao, YH, Postolache, M, Kang, SM: Strong convergence of approximated iterations for asymptotically pseudocontractive mappings. Fixed Point Theory Appl. 2014, 100 (2014)

\section{Submit your manuscript to a SpringerOpen ${ }^{\circ}$ journal and benefit from:}

- Convenient online submission

Rigorous peer review

- Immediate publication on acceptance

- Open access: articles freely available online

- High visibility within the field

- Retaining the copyright to your article 